Internist $2005 \cdot 46: 623-629$ DOI 10.1007/s00108-005-1402-0

Online publiziert: 7. April 2005

(c) Springer Medizin Verlag 2005

Schwerpunktherausgeber

M. Battegay, Base

U. Flückiger, Basel

M. Battegay · U. Flückiger

Klinik für Infektiologie, Universitätsspital Basel, Schweiz

\title{
Der Wert der klinischen Infektiologie
}

nem komplizierten Verlauf betreut, die an einer Infektionskrankheiten leiden oder bei welchen eine Infektionskrankheit differenzialdiagnostisch in Betracht gezogen wird. Leitsymptome für ein initiales Konsilium sind häufig Fieber, unklare Entzündungszeichen (z. B. erhöhtes C-reaktives Protein) oder positive Blutkulturen. Entsprechende Patienten weisen in 50-75\% der Fälle Grundkrankheiten oder Risikofaktoren auf und sind auf internistischen Stationen, Intensivpflegeeinheiten, hämatologischen Stationen für autolog und allogen stammzelltransplantierte Patienten mit malignen hämatologischen Grunderkrankungen oder orthopädischen Stationen (z. B. Patienten mit einer Osteomyelitis nach Fremdkörperimplantation) hospitalisiert. Seitens der chirurgischen Patienten sehen wir Erkrankte mit Komplikationen, z. B. nach Abdominaleingriffen oder Traumata. Nach wie vor können HIV-infizierte Patienten eine erhöhte Infektionsrate aufzeigen, die einen Krankenhausbehandlung verlangt.

Insgesamt sind kritische Situation häufig, die kompetente Entscheidungen hinsichtlich diagnostischen und/oder operativen Eingriffen erfordern. Vielschichtige Situationen bestehen bei Katheterinfekten (s. Artikel Widmer in dieser Ausgabe). Hier stellen sich Fragen hinsichtlich Katheter belassen oder entfernen, Typ der Bakterien oder Pilz, Resistenzmuster, korrekte Auswahl, Dauer und Applikation eines Antibiotikums.

In - Abbildung 1 sind zusätzliche Aufgaben einer Infektiologie aufgezeichnet, namentlich das Antibiotic Stewardship
Programm (s. unten), die ambulante HIVSprechstunde sowie die enge Zusammenarbeit mit einer Abteilung für Spitalhygiene und mit diagnostischen Labors. Selbstverständlich variiert die Struktur von Klinik zu Klinik und spezifische Bereiche können selbständig integriert oder in anderen Kliniken eingebunden sein.

\section{Infektiologische Konsilien bei hospitalisierten Patienten}

Essenziell ist es, Patienten im Längsverlauf während des gesamten Krankenhausaufenthalts zu beurteilen. Bei der initialen Beurteilung (infektiologisches Erstkonsilium) muss der Patient vom infektiologischen Konsiliararzt befragt und untersucht werden. Die Information muss mit zusätzlicher Information der direkt betreuenden Ärzte und Pflegenden ergänzt werden (z. B. wie war das operative Prozedere?, wo liegen Drainagen?, sind Fremdkörper eingesetzt worden?, wann und wie wurde eine Biopsie entnommen?). Am gleichen Tag erfolgt eine mitunter detektivische Suche nach weiteren Informationen in Krankengeschichten und Krankenkurven (wie ist der genaue Fieberverlauf?, liegt ein Bakteriologieresultat vor?, sind Antibiotika bereits verabreicht worden? etc.).

Im Rahmen unserer Klinik findet eine tägliche 60 min dauernde Besprechung mit den Ärzten des Konsiliardienstes (Assistenzarzt, Oberarzt, Leiter), den Mikrobiologen und den Spitalhygienikern statt. Dabei werden alle klinischen und radiologischen Daten vorgestellt und mit den entsprechenden bakteriologischen und se-
Im Konsiliardienst werden schwerkranke Patienten oder Patienten mit ei- 


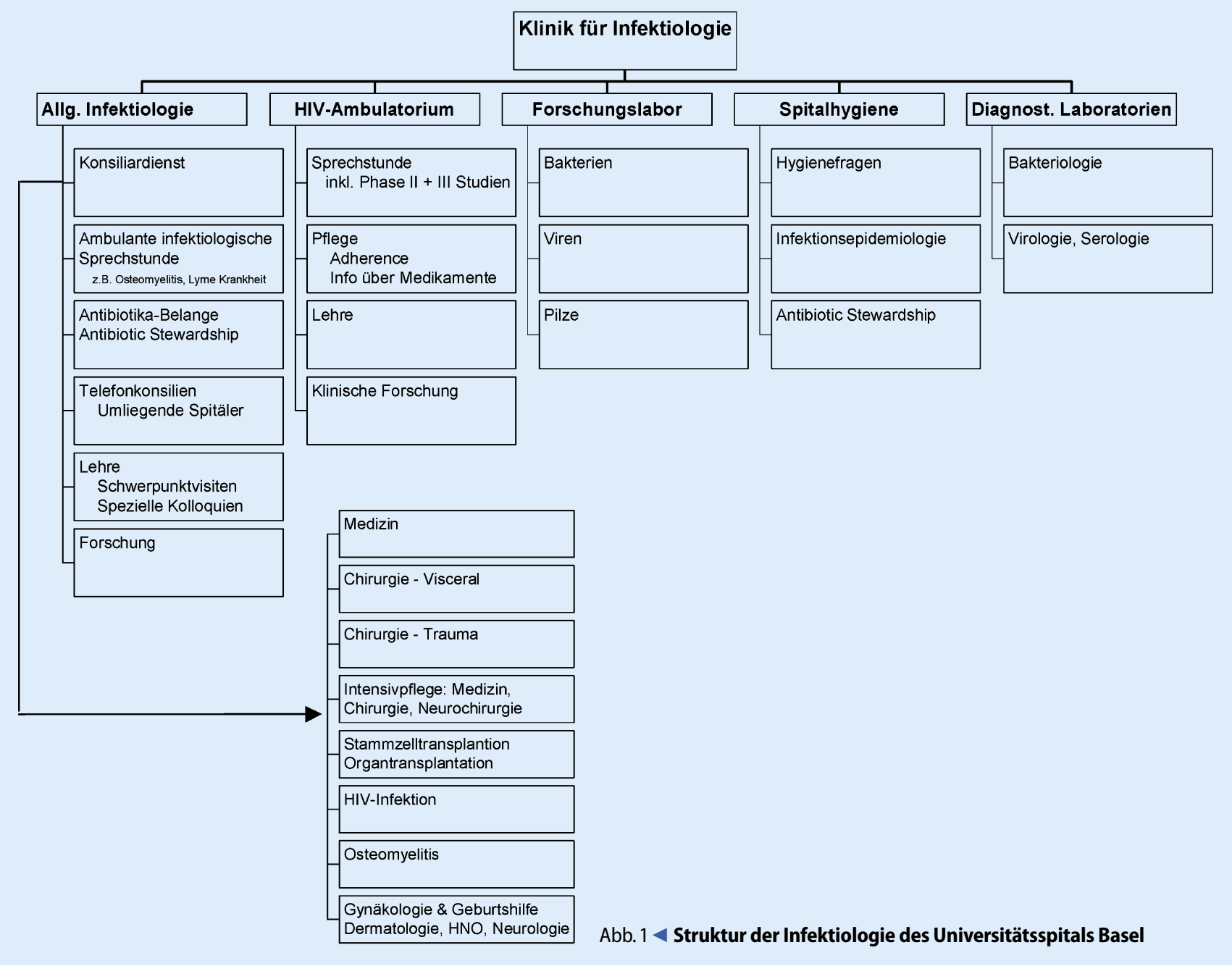

rologischen Resultaten der Patienten, bei denen ein Konsilium stattfand, diskutiert. Das weitere sinnvolle diagnostische und therapeutische Vorgehen wird besprochen, entschieden und schriftlich festgelegt. Für eine möglichst korrekte Beurteilung ist die klinische Untersuchung (d. h. Konsilien vor Ort) unerlässlich, weshalb der Assistenzarzt den Patienten vor der Besprechung untersucht hat.

Weiter müssen bei Entscheidungen einer empirischen Antibiotikagabe die Kenntnisse der lokalen Resistenzlage berücksichtigt werden. So muss z. B. in Basel bei Verdacht eines S.-aureus-Infekts kein Vancomycin gegeben werden, da wir praktisch keine nosokomialen MRSA-Infektionen (methicillinresistente S. aureus) haben. Hingegen muss MRSA mitbehandelt werden, falls Patienten aus anderen Spitälern mit hoher MRSAPrävalenz verlegt werden.

Die Interpretation der Bedeutung der isolierten Mikrooganismen kann schwie- rig sein. Die Entscheidung über Infektion, Kolonisation oder Kontamination ist oft nur nach Durchsicht der klinischen Daten in Zusammenhang mit den mikrobiologischen Resultaten möglich, häufig sogar erst im Verlauf des stationären Aufenthalts oder nach zusätzlichen Abklärungen. Deshalb ist die mehrtägige klinische Verlaufsbeobachtung (Folgekonsilien) durch den Infektiologen notwendig. Sobald das Resistenzmuster der Keime im Verlauf bekannt ist, sollte der klinische Infektiologe einen Vorschlag für eine gezielte antibiotische Therapie machen, bzw. sicherstellen, dass der Patient ein wirksames Antibiotikum erhält.

Hier sind wiederum infektiologisch-klinische Kenntnisse notwendig. Nicht jedes im Antibiogramm erscheinende empfindliche Antibiotikum ist auch ein bei der entsprechenden Infektion korrektes Antibiotikum.

\section{- Die Infektion entscheidet über die adäquate Antibiotikatherapie.}

So würde z. B. bei einem Patienten mit positiven Blutkulturen mit Enterococcus faecalis, der empfindlich ist auf Penicillin, Ciprofloxacin, Vancomycin und „High-levelGaramycin“, eine Therapie mit Ciprofloxacin falsch sein. Zudem muss bei positiven Blutkulturen mit diesem Keim eine Endokarditis klinisch gesucht und ausgeschlossen werden. Erst dann kann eine Empfehlung zur Antibiotikatherapie und Dauer gegeben werden. Dieses Beispiel zeigt, dass der klinische Infektiologe täglich präsent sein muss und die Konsilien innerhalb weniger Stunden nach Anfrage gemacht werden müssen.

Eine weiteres Beispiel sind positive Blutkulturen mit Enterobakterien, die eine induzierbaren ampC-Resistenz aufweisen. Solche Bakterien dürften nicht mit Cephalosporinen der 3. Generation behandelt werden, obwohl diese im Antibiogramm sensibel reagieren. Dies zeigt, dass der klinische Infektiologe eng mit dem bakterio- 


\section{Hier steht eine Anzeige.}

黛 Springer 
Tabelle 1

Indikationen für ein infektiologisches Konsil

\begin{tabular}{|c|c|c|c|}
\hline Grundkrankheit & 1. Problem & Komplikation & Interdisziplinäre Gruppe aus \\
\hline Diabetes mellitus & Diabetischer Fuß & Knocheninfektion & $\begin{array}{l}\text { Infektiologie, Mikrobiologie, Chirurgie, Innere Medizin, } \\
\text { Endokrinologie, Angiologie }\end{array}$ \\
\hline Koronare Herzkrankheit & Aortokoronarer Bypass & Sternuminfektion & Infektiologie, Mikrobiologie, Chirurgie \\
\hline Stammzelltransplantation & Fieber & Z. B. Aspergillen & Infektiologie, Mikrobiologie, Hämatologie \\
\hline Multimorbidität & Sepsis, Pleuraempyem & Z. B. Pneumokokken & Infektiologie, Mikrobiologie, Pneumologie, Innere Medizin \\
\hline
\end{tabular}

Tabelle 2

Häufigste Fragestellungen bei

Konsilien 2004 bei hospitalisierten Patienten ( $\mathrm{n=2942)}$

\begin{tabular}{|lll}
\hline Fragestellung & $\mathbf{n}$ & [\%] \\
\hline $\begin{array}{l}\text { Osteomyelitis (inkl. 141 } \\
\text { Fremdkörperinfektionen) }\end{array}$ & 497 & 16,9 \\
\hline Sepsis & 479 & 16,3 \\
\hline Fieber & 395 & 13,4 \\
\hline Pneumonie/Bronchitis & 246 & 8,4 \\
\hline Abszess & 171 & 5,8 \\
\hline Entzündungszeichen & 149 & 5,1 \\
\hline Cellulitis/Erysipel & 99 & 3,4 \\
\hline Wundinfekt & 91 & 3,1 \\
\hline Meningitis/Enzephalitis & 88 & 3,0 \\
\hline Abdominalinfekt & 72 & $<3$ \\
\hline Tuberkulose & 69 & $<3$ \\
\hline Endokarditis & 54 & $<3$ \\
\hline Harnwegsinfekt/ & 50 & $<3$ \\
\hline Pyelonephritis & & \\
\hline HIV & 25 & $<3$ \\
\hline Arthritis & 14 & $<3$ \\
\hline
\end{tabular}

logischen Labor zusammenarbeiten und über gute Fachkenntnisse der Mikrobiologie verfügen sollte. Entscheidend ist, dass bei infektiologischen Konsilien das Wissen des klinischen Bildes einer bestimmten Infektionskrankheit und der isolierten Mikrorganismen zusammen interpretiert werden müssen. Es wäre falsch, Bakterien isoliert ohne das dazu passende klinische Bild zu behandeln.

\section{Beurteilung von Blutkulturen}

Zusätzlich zu den als Auftrag gegebenen Konsilien werden uns sämtliche positive Blutkulturen des gesamten Spitals täglich mitgeteilt. Gerade bei der Isolation von koagulasenegativen Staphylokokken muss zwischen Infektion und Kontamination unterschieden werden. Angaben des Mikrobiologen über Anzahl der positiven Blutkulturen und der Inkubationszeit erlauben eine erste Interpretation, die dann bei einer Durchsicht der Krankengeschichte und Patientenuntersuchung meistens geklärt werden kann.

Mit diesem Konzept lassen sich unnötige Vancomycingaben (da koagulasenegative Staphylokokken häufig oxacillinresistent sind, wird meisten ein Glykopeptid verabreicht) z. B. bei Patienten auf der Intensivstationen vermeiden. Jedoch erlaubt auch ein aktives Nachfragen bei den zuständigen Ärzten von Patienten mit positiven Blutkulturen über Infektionsfokus und Therapien, falsche Therapien zu finden und zu korrigieren.

\section{Interdisziplinäre Arbeit}

Als kontinuierliche Weiterbildung für ein spezifisches infektiologisches Teaching werden einmal pro Woche 20-minütige Schwerpunktvisiten auf verschiedenen Kliniken mit häufiger Infektionsfrequenz abgehalten (z. B. Intensivpflegestationen; dies geschieht anhand von Vorstellungen stationärer Patienten) oder es werden einmal pro Woche gemeinsame Visiten durchgeführt (z. B. bei stammzelltransplantierten Patienten).

In der - Tabelle 1 sind weitere Beispiele genannt, die Probleme aufzeigen, bei denen ein infektiologisches Konsilium hilfreich sein kann. Insbesondere zeigen diese Beispiele auch die Wichtigkeit der interdisziplinären Arbeit. Meistens besteht ein Grundproblem (z. B. Patienten mit Diabetes mellitus und einem diabetischen Fuß, Patienten nach aortokoronarem Bypass, usw.). Der Verlauf zeigt im Weiteren eine vermutete oder bereits diagnostizierte Komplikation an, im Falle des diabetischen Fuß die begleitende Osteomyelitis oder beim Patienten nach aortokoronarem Bypass die beginnende Sternuminfektion. In der Folge ist häufig ein interdisziplinäres Vorgehen nötig. So muss bei beiden oben beschriebenen $\mathrm{Pa}$ tienten ein chirurgischen Débridement (Chirurge) erfolgen mit Knochenbiopsien, die histologisch (Pathologe) und bakteriologisch (Mikrobioolge) untersucht werden. Die Signifikanz der gefundenen Keime (Kontamination/Kolonisation/Infektion) muss dann in einem interdisziplinären Gespräch mit behandelndem Arzt, Infektiologen, Chirurgen, Mikrobiologen und je nach Patienten andere Fachspezialisten diskutiert und das weitere Prozedere festgelegt werden.

Im Rahmen der über 2900 im Jahre 2004 durchgeführten Konsilien am Universitätsspital Basel zeigt • Tabelle 2, dass die Sepsis und ein unklarer Fieberzustand die häufigsten Gründe für ein Konsilium sind. Daraufhin folgt die Pneumonie und Bronchitis. Anzumerken ist, dass wir selten zu einem Patienten mit Pneumonie auf der Notfallstation gebeten werden, wo meistens eine klarere Situation vorhanden ist. Bei Konsilien wegen Pneumonie handelt es sich um beatmete Patienten oder Patienten, die einen komplizierten postoperativen Verlauf aufweisen, z. B. nosokomiale Pneumonien.

Entsprechend der Krankheitsbilder werden Konsilien von Infektiologen in etwa der Hälfte der Fälle auf der Medizin (50\% in unserem Spital) durchgeführt. Einen relativ hohen Anteil nimmt dabei die Isolierstation für Patienten mit hämatologischen Grundleiden sowie die medizinische Intensivstation ein. Ein großer Teil der Konsilien wird auf der Chirurgie durchgeführt (40\%), weitere auf anderen Kliniken wie Gynäkologie, Dermatologie, Neurologie 
und der interdisziplinären Notfallstation. Mehrere Studien haben gezeigt, dass ca. 610\% aller Spitalpatienten von Infektiologen gesehen werden $[1,2]$. Im Vordergrund der Arbeit der Infektiologen steht in diesen Studien, wie in unserem Spital, die initiale Beurteilung und Antibiotikatherapie sowie die Unsicherheit bei Diagnostik und Therapie, bei nicht Ansprechen auf Antibiotika. Häufig genannte Gründe eines Konsiliums waren atypische Manifestationen einer häufigen Krankheit, unklares Fieber und klinische Verschlechterung trotz vermeintlich adäquater Therapie.

\section{Studien zum Einfluss der Infektiologie}

Die Wirkung infektiologischer Konsilien hängt von der Adhärenz über Empfehlungen ab [3]. In einer Arbeit, welche 465 Konsilien untersuchte, bezogen sich die Hälfte auf die Therapie (49\%), 10\% auf Therapie und Diagnose und die restlichen auf Diagnose und Prävention. Hinsichtlich der Therapie war die adäquate Wahl eines Antibiotikums die wichtigste konsiliarische Empfehlung. Häufig sind Algorithmen und Empfehlungen für spezifische Situationen nicht vorhanden. Der Grund liegt darin, dass die Komplexität bei Multimorbidität, das einfache Anwenden dieser Algorithmen zumindest in Frage stellt. Nichtinfektiologen können vielfach infektiologische Diagnosen und Therapien stellen, respektive durchführen. Trotzdem bleibt es dem Infektiologen überlassen, in einer kurzen Zeitspanne mit wenig Ressourcen schwierige Diagnosen zu stellen und spezifische Therapien vorzuschlagen.

Folglich war die Adhärenz an infektiologischer Konsilien mit 80\% hoch und noch höher bei kritischen Empfehlungen (85\%) wie invasiven diagnostischen Eingriffen oder Therapieänderungen bei schwerkranken Patienten [3, 4]. Therapeutische Empfehlungen wurden eher befolgt als diagnostische Empfehlungen. Ebenfalls wichtig ist eine gute Lesbarkeit und eine schnelle Abgabe von Konsilien [3]. Gründe für die Hochschätzung infektiologischer Konsilien sind unserer Erfahrung nach die Gründlichkeit, die fundierten klinischen und diagnostischen Fähigkeiten, der detaillierte Bericht und der zuverlässige Follow-up, d. h. die Mitbetreuung der Patienten [4].

Internist 2005 • 46:623-629

DOI 10.1007/s00108-005-1402-0

○) Springer Medizin Verlag 2005

\section{Battegay · U. Flückiger}

\section{Der Wert der klinischen Infektiologie}

\section{Zusammenfassung}

Infektiologen nehmen in der Betreuung von Patienten mit meist schweren Infektionen für die Diagnostik und Therapie, namentlich der Antibiotikaverordnung eine wichtige Rolle ein. In mittelgroßen und großen Krankenhäusern der Tertiärversorgung, insbesondere Universitätskliniken werden 6-10\% aller hospitalisierten Patienten durch Infektiologen mitbetreut. In den letzten Jahren und Jahrzehnten ist die Morbidität und Mortalität schwerer Infektionen, z. B. der Septikämien, deutlich gesunken. Die Verbesserung der Prognose schwerer Infektionen ist insbesondere durch ei-

\section{Effects of clinical infectiology}

\section{Abstract}

Infectious diseases specialists are involved in patient care mostly regarding patients with severe infections in complex situations. Morbidity and mortality of severe infections such as sepsis has been significantly reduced during past years and decades. Prognosis of severe infections is dependent upon timely and correct diagnosis and appropriate therapy. The diagnostic and therapeutic approach is often invasive and interdisciplinary. Importantly, thoughtful use of antibiotics may increase its effectivity, reduce antibiotic use and occurrence ne zeitgerechte Diagnostik und ein adäquates Management bedingt, das nicht selten invasiv ist, z. B. in Form eines chirurgischen Débridement. Krankheitsbilder sind häufig komplex und machen eine interdisziplinäre Arbeit erforderlich. Dieser Artikel beschreibt die an den Infektiologen gestellten Herausforderungen in einem interdisziplinären Umfeld.

\section{Schlüsselwörter}

Infektiologie · Infektionskrankheiten Antibiotika $\cdot$ Sepsis of bacterial resistance and increase cost effectiveness. Hence, antibiotic management and stewardship has become more and more the focus of work. This article highlights the clinical work of infectious diseases specialists in an interdisciplinary setting and describes there value to patients and hospitals.

Keywords Infectious diseases · Antibiotics · Sepsis . Bacterial resistance 
Eine infektiologische Expertise hat bei Patienten mit Staphylococcus-aureus-Sepsis [5], mit HIV-Infektion [6, 7], mit Osteomyelitis [8] und bei Patienten mit Pneumokokkensepsis [9] einen günstigen prognostischen Einfluss.

\section{Infektiologische Erfolge bei der Sepsis}

Die Mortalität der Sepsis nahm in den letzten 2-3 Dekaden drastisch ab $[5,10,11$, $12,13,14]$. In einer eigenen Studie wurden 103 Patienten mit einer Sepsis untersucht. Infektiologen änderten die Therapie häufiger von einem Breitspektrum- zu einem spezifischeren Antibiotikum, wenn das Resultat der Blutkulturen bekannt wurde [15]. Ebenfalls war die empirische Therapie häufig adäquater als bei Nichtspezialisten.

In einer großen weiteren Studie, die am Universitätsspital Basel durchgeführt wurde, zeigte sich, dass die Sterblichkeit von Patienten mit einer Pneumokokkensepsis signifikant von der ersten (1986-1993) zur zweiten Periode (1993-200o) abnahm. Die Abnahme der Sterblichkeit war mit $33 \%$ auf $17 \%$ eindrücklich ( $\mathrm{p}<0,001$; [9]). Die meisten Patienten wiesen mehrere, mitunter schwere Grunderkrankungen oder Risikofaktoren wie chronische Lungenkrankheiten, Tumore, Alkoholismus, Diabetes mellitus, eine immunsuppressive Therapie, HIV-Infektion, Splenektomie oder Neutropenie auf. Die Autoren führten 3 Gründe an, wie es zu einer Reduktion der Sepsis kommen konnte. Erstens wurde die Abteilung für Infektiologie mit einem entsprechenden Konsiliardienst 1991 gegründet und war ab 1994 spitalweit tätig. Der Konsiliardienst empfahl seither den optimalen Diagnoseweg und eine optimale antimikrobielle Therapie. Die anderen beiden genannten Faktoren waren eine möglicherweise reduzierte Pneumokokkenvirulenz sowie die Abnahme der Prävalenz von intravenösen Drogenkonsumenten und von Patienten mit schweren Grunderkrankungen. So waren in der zweiten Periode weniger Patienten mit koronarer Herzkrankheit zu verzeichnen [9]. In dieser Studie hatten 15\% der Patienten 1 Risikofaktor, $23 \% 2$ Risikofaktoren, $27 \% 3$ Risikofaktoren und $29 \%$ hatten 4 oder mehr Risikofaktoren. Nur in 6\% al- ler Episoden fand sich keine Grundkrankheit oder ein Risikofaktor.

In dieser Studie konnte gezeigt werden, dass die Abnahme der Mortalität nicht durch neu auf dem Markt zugelassene Antibiotikatherapien per se Zustande kam, sondern durch die optimalere und vor allem schnellere Verabreichung $[9,16]$. Wie bei der Studie über Pneumokokkensepsis nahmen generell nosokomiale Septikämien hinsichtlich Risiko des Todes ab.

\section{Antibiotic Stewardship}

Ein wichtiges Programm vieler Spitäler ist die sinnvolle und gezielte Abgabe von Antibiotika. Es gibt viele Studien, die die Antibiotikaverschreibepraxis untersucht haben. Es gibt jedoch wenige kontrollierte Studien, die zeigen, dass durch Programme Antibiotika besser verschrieben werden können. Wichtige generelle Elemente der Antibiotic Stewardship sind:

1. das Monitorisieren und ein Feedback über resistente Pathogene (Spitalhygiene),

2. das Wissen, welche Antibiotika wo und wie gebraucht werden [17],

3. die optimale Wahl und Dauer der empirischen Antibiotikatherapie,

4. eine Optimierung der perioperativen antimikrobiellen Prophylaxe.

Beispielsweise konnte gezeigt werden, dass das Überleben bei nosokomialer Pneumonie mit kurzer oder längerer Antibiotikagabe ( 8 vs. 15 Tage) gleich gut war, dass aber in der Gruppe der 15-tägigen Therapie eine signifikant höhere Rate von multiresistenten Pathogenen zu verzeichnen war [18].

Der Antibiotikagebrauch kann durch spezifische Maßnahmen im Rahmen eines Antibiotoc Stewardship Programmes verbessert werden: Verbesserung der Diagnose, Restriktionen hinsichtlich Formulierungen, Verbesserung der Verschreibepraxis durch Weiterbildung, Richtlinien und Algorithmen sowie spezielle Bestellformulare. In einem systematischen Review von 40 Studien [19] zeigte sich, dass lokal adaptierte Guidelines, interaktive Weiterbildung in kleineren Gruppen mit Teilnahme von „Opinion Leaders“ sowie einem Feedbacksystem die besten Resultate aufwiesen. Diese Aspekte betonen, dass ein ausgezeichneter mikrobiologischer Support essenziell ist und eine Verzögerung von Resultaten die klinische Infektiologie entscheidend schwächen würde.

\section{Ambulante Infektiologie}

Wegen potenter und vereinfachter antiretroviraler Therapien hat die Morbidität und Mortalität der HIV-Infektion um ca. $85 \%$ drastisch abgenommen und Konsilien bei hospitalisierten Patienten sind seltener (• Tabelle 2). Hingegen ist die ambulante Infektiologie in den letzten 20 Jahren durch die HIV-Krankheit und die Betreuung von HIV-infizierten Patienten geprägt. Hier bestehen nach wie vor komplexe diagnostische und pharmakotherapeutische Herausforderungen, die ein Spezialwissen und spezialärztliche Erfahrung nötig machen. Insbesondere sind Therapiewechsel trotz virologischen Ansprechens häufig und eine individualisierte Therapie nötig. An vielen Zentren ist die ambulante HIV-Sprechstunde im Rahmen einer Infektiologie organisiert oder eng mit ihr verbunden. Nach wie vor ist die Forschung im diagnostisch-therapeutischen Bereich sehr dynamisch und wird wohl nur von Ärzten verfolgt, die sich der Betreuung einer Mindestzahl von Patienten widmen (>20-50 Patienten).

\section{Schlussfolgerungen}

Die Infektiologie verfügt bei hospitalisierten und ambulanten Patienten mit entsprechenden Krankheiten und Symptomkomplexen über einen hohen Stellenwert. Die Infektiologie hat gerade in den letzten Jahren eine beträchtliche zusätzliche Bedeutung gewonnen. Dies ist bedingt durch

- eine eindrückliche klinisch-analytische, sehr häufig interdisziplinäre Tätigkeit, v. a. mit der Chirurgie, - wirklich neue Infektionskrankheiten wie z. B. HIV, SARS und Vogelgrippe,

- das Wiederaufkommen alter Infektionen wie der Tuberkulose,

- durch weltweite Ausbreitungsmuster von Infektionskrankheiten mit eindrücklichen Patientengeschichten (z. B. Malaria, Dengue, Rickettsien, Q-Fieber), 
- aufkommende Infektionen bei „neuen“ Patientengruppen wie Transplantierten und HIV-infizierten, die neue Risiken für spezielle Infektionskrankheiten aufweisen.

$\mathrm{Zu}$ erwähnen sind in diesem Zusammenhang neue Medikamente wie Virostatika und Fungistatika. Zu guter letzt sind mit Epidemien soziale und politische Aspekte verknüpft, welche über das Fach und die Medizin hinausgehende Herausforderungen stellen.

\section{Fazit für die Praxis}

Rund 6-10\% aller hospitalisierten Patienten werden in großen Spitälern, v. a. Universitätskliniken durch Infektiologen mitbetreut. Studien haben gezeigt, dass eine infektiologische Expertise bei Patienten mit Staphylococcus-aureus-Sepsis, HIV-Infektion, Osteomyelitis und Pneumokokkensepsis einen günstigen prognostischen Einfluss hat. Eine zeitgerechte Diagnostik und adäquates Management ist nicht selten invasiv, z. B. in Form eines chirurgischen Débridements. Dies verlangt eine infektiologische Initial- und Verlaufsbeurteilung.

Antibiotic Stewardship bedeutet, das Monitorisieren und ein Feedback über resistente Pathogene, das Wissen, welche Antibiotika wo und wie gebraucht werden, und die optimale Wahl und Dauer der Antibiotikatherapie.

\section{Korrespondierender Autor Prof. Dr. M. Battegay}

Klinik für Infektiologie, Universitätsspital, Petersgraben 4, 4031 Basel, Schweiz E-Mail:mbattegay@uhbs.ch

Interessenkonflikt: Der korrespondierende Autor versichert, dass keine Verbindungen mit einer Firma, deren Produkt in dem Artikel genannt ist, oder einer Firma, die ein Konkurrenzprodukt vertreibt, bestehen.

\section{Literatur}

1. Yinnon AM (2001) Whither infectious diseases consultations? Analysis of 14,005 consultations from a 5-year period. Clin Infect Dis 33: 1661-1667

2. Petrak RM, Sexton DJ, Butera ML et al. (2003) The value of an infectious diseases specialist. Clin Infect Dis 36: 1013-1017
3. Lo E, Rezai K, Evans AT et al. (2004) Why don't they listen? Adherence to recommendations of infectious disease consultations. Clin Infect Dis 38: $1212-1218$

4. Tenenbaum MJ (2004) Infectious diseases consultative recommendations: if heard, they can be listened to. Clin Infect Dis 38: 1219-1221

5. Fowler VG, Sanders LL, Sexton DJ et al. (1998) Outcome of Staphylococcus aureus bacteremia according to compliance with recommendations of infectious diseases specialists: experience with 244 patients. Clin Infect Dis 27: 478-486

6. Kitahata MM, Koepsell TD, Deyo RA, Maxwell CL, Dodge WT, Wagner EH (1996) Physicians' experience with the acquired immunodeficiency syndrome as a factor in patients' survival. N Engl J Med 334: 701-706

7. Battegay M, Wirz M, Steuerwald MH, Egger M (1998) Early participation in an HIV cohort study slows disease progression and improves survival. The Swiss HIV Cohort Study. J Intern Med 244: 479487

8. Lobati F, Herndon B, Bamberger D (2001) Osteomyelitis: etiology, diagnosis, treatment and outcome in a public versus a private institution. Infection 29: 333-336

9. Trampuz A, Widmer AF, Fluckiger U, Haenggi M, Frei R, Zimmerli W (2004) Changes in the epidemiology of pneumococcal bacteremia in a Swiss university hospital during a 15-year period, 19862000. Mayo Clin Proc 79: 604-612

10. Doern GV, Vautour R, Gaudet M, Levy B (1994) Clinical impact of rapid in vitro susceptibility testing and bacterial identification. J Clin Microbiol 32: 1757-1762

11. Elhanan G, Sarhat M, Raz R (1997) Empiric antibiotic treatment and the misuse of culture results and antibiotic sensitivities in patients with communityacquired bacteraemia due to urinary tract infection. J Infect 35: 283-188

12. Gomez J, Conde Cavero SJ, Hernandez Cardona JL, Nunez ML, Ruiz Gomez J, Canteras M, Valdes $M$ (1996) The influence of the opinion of an infectious disease consultant on the appropriateness of antibiotic treatment in a general hospital. J Antimicrob Chemother 38: 309-314

13. Byl B, Clevenbergh $P$, Jacobs F, Struelens MJ, Zech F, Kentos A, Thys JP (1999) Impact of infectious diseases specialists and microbiological data on the appropriateness of antimicrobial therapy for bacteremia. Clin Infect Dis 29: 60-66

14. Nathwani D, Davey P, France AJ, Phillips G, Orange G, Parratt D (1996) Impact of an infection consultation service for bacteraemia on clinical management and use of resources. QJM 89: 789-797

15. Fluckiger U, Zimmerli W, Sax H, Frei R, Widmer AF (2000) Clinical impact of an infectious disease service on the management of bloodstream infection. Eur J Clin Microbiol Infect Dis 19: 493-500

16. Maki DG (2004) Pneumococcal bacteremia: lessons learned, yet more to learn. Mayo Clin Proc 79: 599-603

17. Harbarth $S$, Liassine N, Dharan S, Herrault P, Auckenthaler R, Pittet D (2000) Risk factors for persistent carriage of methicillin-resistant Staphylococcus aureus. Clin Infect Dis 31: 1380-1385

18. Chastre J, Wolff M, Fagon JY et al. (2003) Comparison of 8 vs 15 days of antibiotic therapy for ventilator-associated pneumonia in adults: a randomized trial. JAMA 290: 2588-2598

19. Gross PA, Pujat $D$ (2001) Implementing practice guidelines for appropriate antimicrobial usage: a systematic review. Med Care 39: II55-69

\section{August Wilhelm und Lieselotte Becht-Forschungspreis 2005}

Die Deutsche Stiftung für Herzforschung vergibt auch 2005 einen Forschungspreis. Ausgezeichnet wird eine wissenschaftlich hochwertige Forschungsarbeit auf dem Gebiet der Herz-Kreislaufkrankheiten. Teilnahmeberechtigt sind in Deutschland tätige Wissenschaftler bis zum 40. Lebensjahr. Der Preis ist mit 10.000 Euro dotiert. Die Arbeiten dürfen einen Gesamtumfang von 25 Seiten nicht übersteigen und in dieser Form noch nicht veröffentlicht sein. Eine Zusammenfassung in deutscher Sprache ist sowohl englischsprachigen Arbeiten als auch deutschen Texten voranzustellen. Die Bewerbungsunterlagen mit tabellarischem Lebenslauf sind in dreifacher Ausfertigung mit Einverständniserklärung der Co-Autoren bis zum 25. Juli 2005 (Poststempel) an die Deutsche Stiftung für Herzforschung, Vogtstraße 50, 60322 Frankfurt am Main, einzusenden. Der Bewerber verpflichtet sich, im Falle einer Prämierung zusätzlich eine allgemeinverständliche Kurzfassung zu erstellen, die für eine Veröffentlichung in der Zeitschrift der Deutschen Herzstiftung „Herz heute" geeignet ist. Über die Vergabe des Preises entscheidet der Vorstand der Deutschen Stiftung für Herzforschung auf Vorschlag des Wissenschaftlichen Beirates.

Informationen: Deutsche Stiftung für Herzforschung, Valerie Popp, Telefon 069/95 51 28-119, e-mail: popp@herzstiftung.de; Internet: www.herzstifung.de

Quelle: Deutsche Stiftung für Herzforschung. 